Biology | Jennifer Geddes-McAlister

\section{Proteomics reveals new possibilities in the fight against infection}

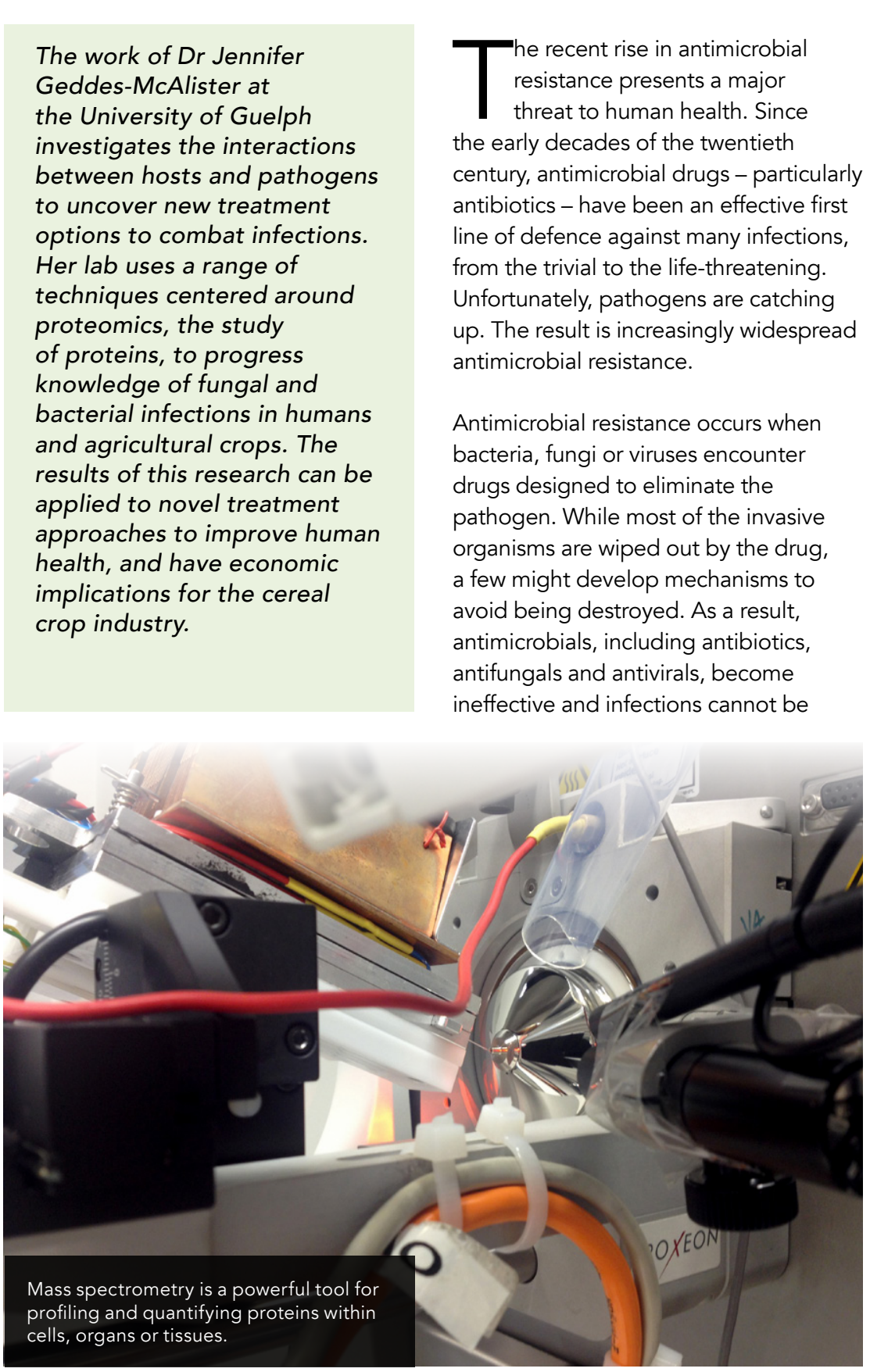

easily treated. The importance of developing alternative strategies highlighted in the World Health Organization's 'Gl Weal tealt 'antimicrobial resistance', which includes aims to improve understanding of drug resistance and optimise the use of current treatments.

As drug resistance spreads, there is a pressing need for new antimicrobial treatments. To develop new drugs, we first need to understand more about how pathogens work. Understanding how pathogens infect, survive and reproduce will allow development of specifically targeted therapeutics can be developed.

\section{DISCOVERY-DRIVEN PROTEOMICS} Dr Jennifer Geddes-McAlister of the University of Guelph is leading research gap. Her work explores the complex interactions between host and

pathogen, with the aim of uncovering new treatment options that can be used in the fight against infection.

Dr Geddes-McAlister uses discoverydriven proteomics, also known as bottom-up proteomics, in her research. Proteomics is the large-scale study of proteins within an organism or within a particular biological setting. This type of research uses a variety of different approaches, including mass spectrometry, which provides a powe tool for profiling and quantitying proteins within cells, organs or tissues. Discovery-driven proteomics begins with proteins. The protein fragments are By identifying wh a mass spectrom protein, the mass spectrometer can infer the existence of specific proteins by piecing together the component parts. In contrast top-down proteomics uses intact proteins to map protein complexes and targeted proteomics is often associated with identification of biomarkers: molecules specific to a unique biological context or organism.

Dr Geddes-McAlister uses discoverydriven proteomics to measure changes in protein levels under different conditions and in various biological systems. Recently, proteomics has developing

TACKLING FUNGAL PATHOGENS VIA PROTEOMICS

fungal pathogens cause a wide

froy of diseases in humans, ranging

The underlying trigger for onset of

infection is often a shift in the immune status of the host; many opportunistic pathogens are harmless until their host is immunocompromised. Although the source and route of infection and resulting symptoms may differ betwe species, there are worryingly high morbidity and mortality rates associated with fungal invasion.

The successful treatment of fungal infections is challenging. Significantly, many of the molecules targeted by human cells. Also, there is a limited section of clinicall effective, non-toxic antifungal agents, and treatment must often be lengthy to be succesfful. $\mathrm{Dr}$ Geddes-McAlister and her colleagues use quantitative proteomics to discover how fungal pathogens interact with their host, and to understand why the host is often unable to clear the infection. This research offers new insight into how fungi cause disease and the mechanisms they use to evade the host immune response.

An interesting example of proteomics in action is the characterisation of a human fungal pathogen called Cryptococcus neofors which is

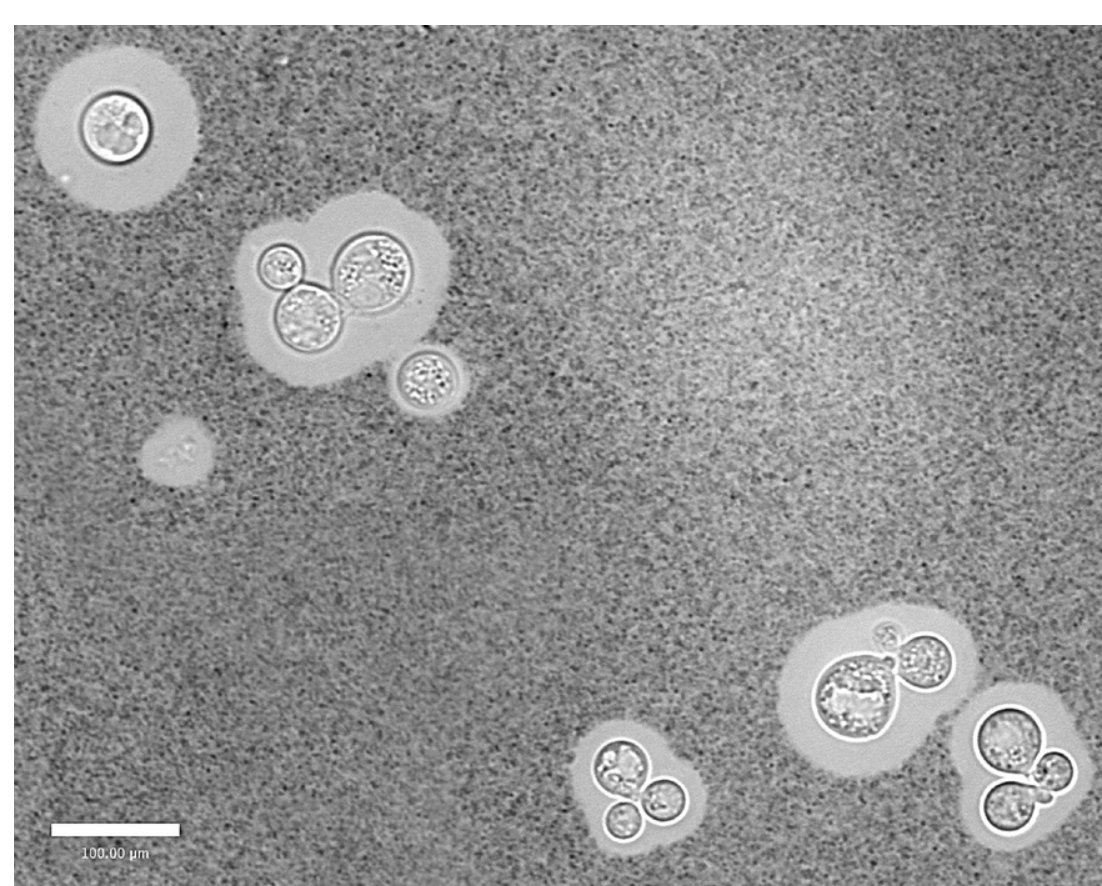

Microscopy image of Cryptococcus

The rise in antimicrobial resistance presents a major threat to human health.

in inmunocompromised individuals. Historically, study of C. neoform techniques, such as genomic analyses. However, recent advances in mass spectrometry and bioinformatic tools and databases offer an opportunity to understand more about the biological processes used by fungi during infection, such as the release or provides insight into the regulatory mechanisms critical to disease.

Given the growing emergence of antifungal-resistant strains of $C$. neoformans, learning more about proteins produced in response to routes. Dr Geddes-McAlister has recently both described useful ways to prepare $C$. neoformans samples, and designed protocols for profiling changes in the proteome. This provides a grounding for future studies to take dis work further, one day leading to a deeper understanding of how and why frungal infection.

The overall goal of Dr GeddesMcAlister's research is to identify new strategies to disrupt the interaction between host and pathogen, thus eliance on antifungals as

\section{BACTERIAL PATHOG}

AND BIOMARKERS

Dro hel to tackle oth research can Some bacteria such as Pseudomon aeruginosa, produce antibiotic-resistant biofilms-a phenomenon where cells stick both to each other and to a surface. In the case of $P$. aeruginosa this ability protects the bacteria from destruction by antibiotics commonly used to treat eye infections. Dr GeddesMcAlister and her team recently used quantitative proteomics to successfully identify biomarkers of $P$. aeruginosa infection of the eye surface, as well as to distinguish between general and site-specific host responses to bacterial infection. The ability to recognise patecules specific to a particular pathogen can be an important step diagnostic tests. 


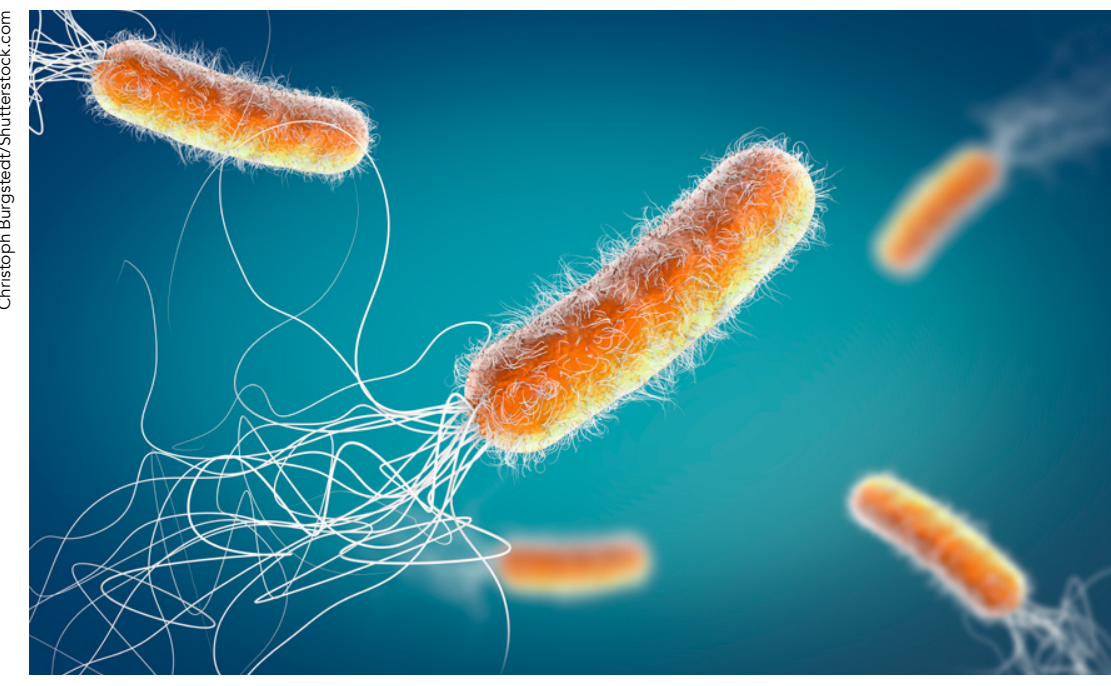

Dr Geddes-McAlister's research blends cutting-edge proteomics technologies with a systems
biology perspective to develop novel therapeutics.

Dr Geddes-McAlister uses discoverydriven proteomics to measure changes in protein levels under different conditions and in various biological systems.

Mass spectrometry-based proteomics has also been employed to investigate the relationship between iron availability and Klebsiella pneumoniae, a bacterium which usually lives in the gastrointestinal tract but which can cause severe infectio if spread to other parts of the body. Dr Geddes-McAlister and her research group uncovered a new role of an enzyme called Lon protease in promoting iron balance in bacterial cells. Iron is an essential component of $K$. pneumoniae survival. Undrients - like mon about how limiting responses could be key to developing responses could be
future antibiotics.

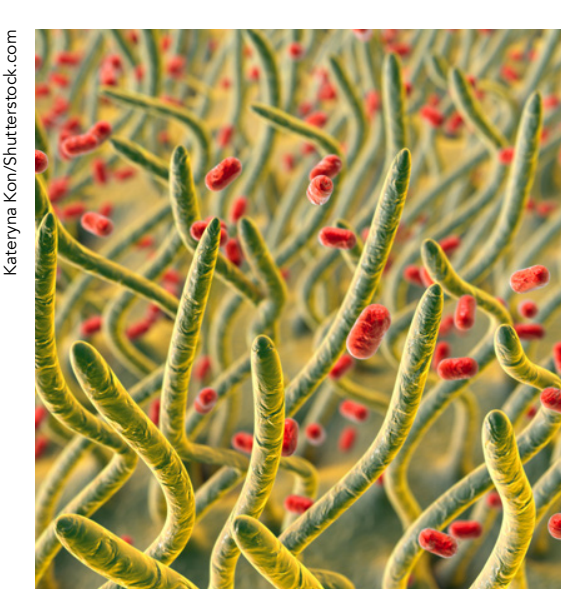

PROTECTING CROPS

THROUGH PROTEOMICS

The versatile techniques used by $D$

Geddes-McAlister and her research

group can also be applied to agricultura settings. For example, quantitative proteomics can be used to describe the interactions between environmental fun and economically important crops. The fungus Fusarium graminearum causes the condition called Fusarium wheat and barley. This disease, which can give crops a shrivelled bleached appearance, leads to the accumulation

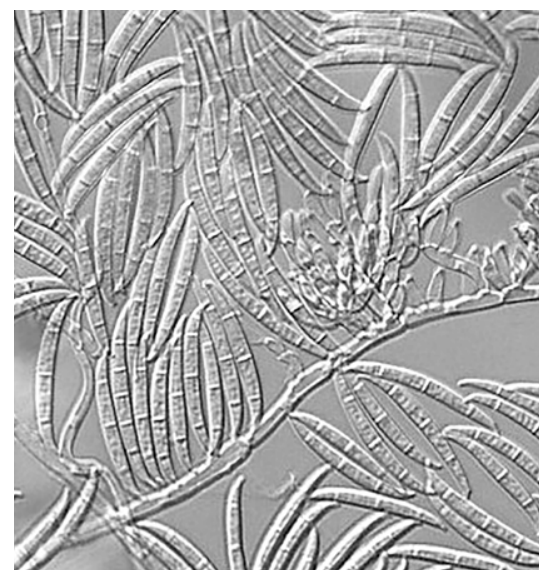

Mass spectrometry-based proteomics were
used to investigate the relationship between

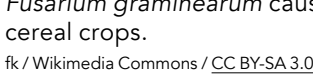

of mycotoxins (fungal toxins) in the sizes and dects, severely reduced crop be devastating. Infected crops cannot be sold, causing substantial economic loss. However, if these damaged grain do make it into animal feed, the toxins can be harmful to livestock and poulty Mycotoxins can also seep into ground water and present a significant threat to human health.

Currently, Fusarium head blight is reated with single dosing antifungals, but this is expensive for growers and provides minimal protection against the accumulation of mycotoxins. Dr Geddes McAlister's team are investigating potential new treatments using a system biology approach, including multiple proteomics, such as quantitative (the study of metabolites, the smallmolecules involved in me, the small

These cutting-edge methods will allow the team to identify and characterise mechanisms by which plants clear funga infections and degrade mycotoxins. Specifically, they will study how some crop species are able to survive exposure to $F$. graminearum, and how these plants defend against accumulation of mycotoxins. An improved understanding of both the mechanisms used by crops to protect against infection, and of the ways in which fungal pathogens invade their hosts, may lead to the discovery of genetic markers that convey resistance to disease. These makkers could then breeding strategies to improve yield and quality

\section{THE IMPACT OF DISCOVERY.}

DRIVEN PROTEOMICS

The expertise of Dr Geddes-McAlister

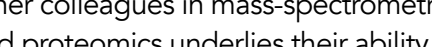
to apply this technology to a plethora of biological questions. Her team are currently investigating host-pathogen interactions with medical, agricultural and economic importance, with the ultimate goal of discovering new therapeutic options and treatments. Increased understanding of these interactions-and the development of new treatments-wil prevent the evolution of drug-resistant pathogens, improve ability to clear and quality of life on a global scale.

\section{Behind the Research}

Dr Jennifer Geddes-McAlister

E: igeddesm@uoguelph.ca $\quad$ T: +1-519-824-4120 ext. 52129 - @ @JGMProteomics
W: https://www.uoguelph.ca/mcb/people/dr-iennifer-geddes-mcalister

Research Objectives Jennifer Geddes-McAlister's research group blends cutting-edge proteomics technologies with a systems biology
perspective to develop novel therapeutics.

\section{Detail}

Jennifer Geddes-McAlister Stone Rd. E., Guelph, Ontario,

Bio of Biochemistry in Germany as an Alexander von Humboldt scholar.

Dr Jennifer Geddes-McAlister joined the University of Guelph as Assistant Professor in July 2018. Previously, she completed a BSc and MSc at the by a PhD at the University of British Columbia, and a post-doctoral
fellowship at the Max Planck Institute

Funding

- Natural Sciences and Engineering Research Council of Canada Discovery Grant - Banting Research Foundation - Univcovery Award - Canadian Foundation for Innovation - Mohn R. Evans Leaders Fund)
Mason Research Fund

References

Ball, B., \& Geddes-McAlister, J. (2019). Quantitative proteomic profiling of C. neoformans. Curr Protocol.

Muselius, B., Sukumaran, A Yeung, I \& Geddes McAlister, J. (2020). Iron limitation in K. pneumoniae defines new roles for Lon protease in homeostasis and degradation by quantitative proteomics. Front.

Yeung, J., Gadjeva, M. \& Geddes-McAlister, J. (2020). Label-Free Quantitative Proteomics Distinguishes General and Site-Specific Host Responses to P. aeruginosa Infection at 2/ Ocular Surface. Proteomics, 20, 190029
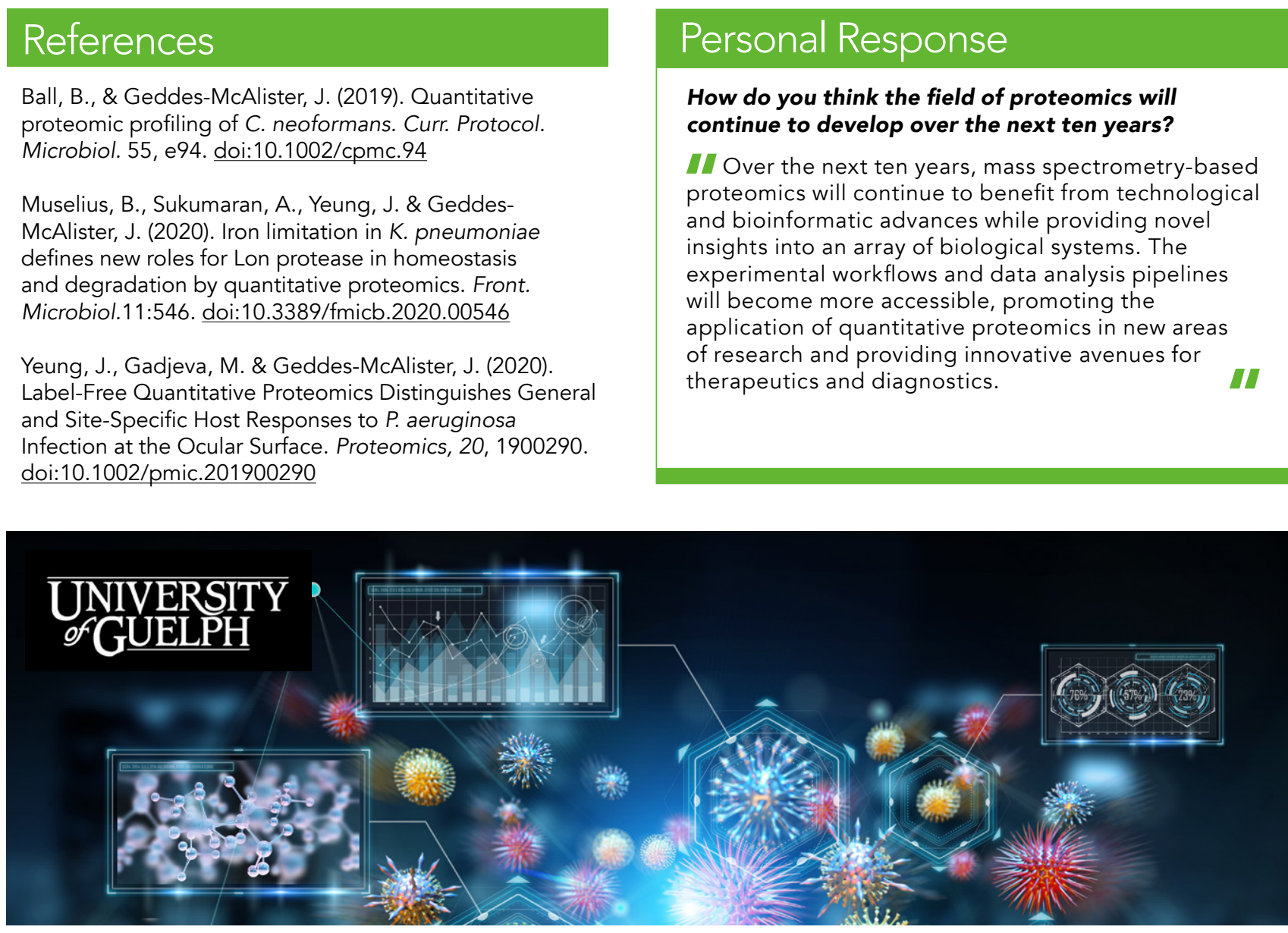